\title{
A Pharmacokinetic Natural Product-Disease-Drug Interaction: A Double Hit of Silymarin and Nonalcoholic Steatohepatitis on Hepatic Transporters in a Rat Model
}

\author{
Michelle L. Montonye, Dan-Dan Tian, Tarana Arman, Katherine D. Lynch, Bruno Hagenbuch, \\ (1) Mary F. Paine, and John D. Clarke
}

Department of Pharmaceutical Sciences, Washington State University, Spokane, Washington (M.L.M., D.-D.T., T.A., K.D.L., M.F.P., J.D.C.) and Department of Pharmacology, Toxicology and Therapeutics, The University of Kansas Medical Center, Kansas City, Kansas (B.H.)

Received May 31, 2019; accepted August 14, 2019

\begin{abstract}
Patients with nonalcoholic steatohepatitis (NASH) exhibit altered hepatic protein expression of metabolizing enzymes and transporters and altered xenobiotic pharmacokinetics. The botanical natural product silymarin, which has been investigated as a treatment of $\mathrm{NASH}$, contains flavonolignans that inhibit organic anion-transporting polypeptide (OATP) transporter function. The purpose of this study was to assess the individual and combined effects of NASH and silymarin on the disposition of the model OATP substrate pitavastatin. Male Sprague Dawley rats were fed a control or a methionine- and choline-deficient diet (NASH model) for 8 weeks. Silymarin $(10 \mathrm{mg} / \mathrm{kg})$ or vehicle followed by pitavastatin $(0.5 \mathrm{mg} / \mathrm{kg})$ were administered intravenously, and the pharmacokinetics were determined. NASH increased mean total flavonolignan area under the plasma concentration-time curve $\left(\mathrm{AUC}_{0-120 \mathrm{~min}}\right)$ 1.7-fold. Silymarin increased pitavastatin $\mathrm{AUC}_{0-120} \mathrm{~min}$ in both control and NASH animals approx. 2-fold. $\mathrm{NASH}$ increased pitavastatin plasma concentrations from 2 to 40 minutes, but $A_{U} C_{0-120}$ min was unchanged. The combination
\end{abstract}

of silymarin and NASH had the greatest effect on pitavastatin $\mathrm{AUC}_{0-120 \mathrm{~min}}$, which increased 2.9-fold compared with control vehicle-treated animals. NASH increased the total amount of pitavastatin excreted into the bile 2.7-fold compared with control animals, whereas silymarin decreased pitavastatin biliary clearance approx. 3-fold in both control and NASH animals. This double hit of NASH and silymarin on hepatic uptake transporters is another example of a multifactorial pharmacokinetic interaction that may have a greater impact on drug disposition than each hit alone.

\section{SIGNIFICANCE STATEMENT}

Multifactorial effects on xenobiotic pharmacokinetics are within the next frontier for precision medicine research and clinical application. The combination of silymarin and NASH is a probable clinical scenario that can affect drug uptake, liver concentrations, biliary elimination, and ultimately, efficacy and toxicity.

\section{Introduction}

Nonalcoholic steatohepatitis (NASH) is a severe form of nonalcoholic fatty liver disease (NAFLD) (Chalasani et al., 2018). An emerging concern for NASH patients is altered expression and function of drug-metabolizing enzymes and transporters, potentially leading to altered pharmacokinetics of xenobiotics. For example, previous studies suggested that decreased sinusoidal expression of hepatic organic anion-transporting polypeptide (OATP) uptake transporters may be responsible for the net 1.4-fold increase in

This work was supported in part by the National Institutes of Health National Institute of Environmental Health Sciences [Grant R00ES024455], the NIH National Center for Complementary and Integrative Health [Grant U54AT008909], the National Institute of General Medical Sciences [Grant R01GM077336], and the Washington State University College of Pharmacy and Pharmaceutical Sciences.

https://doi.org/10.1124/jpet.119.260489. systemic concentrations of ${ }^{99 \mathrm{~m}} \mathrm{Tc}$-mebrofenin observed in NASH patients (Ali et al., 2017; Clarke et al., 2017). In addition, impaired canalicular localization of the efflux transporter multidrug resistance-associated protein (MRP)2 and increased sinusoidal expression of MRP3 may be responsible for higher plasma retention of morphine glucuronides in rodent models of NASH and in NASH patients (Hardwick et al., 2011, 2012, 2013; Dzierlenga et al., 2015; Ferslew et al., 2015). These changes in xenobiotic disposition may alter therapeutic and adverse drug responses in NASH patients.

The high clinical burden of NAFLD has spurred investigation of novel therapeutic agents, including the botanical natural product silymarin. Silymarin, an extract prepared from the seeds of milk thistle [Silybum marianum (L.) Gaertn.] as well as other milk thistle preparations have been used for centuries to treat liver and gallbladder disorders (Abenavoli et al., 2010). Milk thistle has remained within the top 20 best-selling herbal dietary supplements in

ABBREVIATIONS: ANOVA, analysis of variance; AUC, area under the plasma concentration-time curve; BCRP, breast cancer-resistance protein; $\mathrm{CHO}$, Chinese hamster ovary; $\mathrm{Cl}$, systemic clearance; DP, declustering potential; NAFLD, nonalcoholic fatty liver disease; NASH, nonalcoholic steatohepatitis; OATP, organic anion-transporting polypeptide; $\mathrm{V}_{\mathrm{ss}}$, apparent volume of distribution at steady state. 
the U.S. mainstream multioutlet channel for the past 30 years, with 16.8 million dollars in sales in 2017 (Smith et al., 2018). Evidence for the efficacy of silymarin in NAFLD patients is limited, although incorporation of silymarin into treatment plans, either alone or in combination with other agents, continues to be investigated (Abenavoli and Bellentani, 2013). Multiple studies have demonstrated that silymarin is well tolerated at doses greater than one gram, and in NAFLD patients specifically silymarin doses of $700 \mathrm{mg}$ three times daily have been used safely (Hawke et al., 2010; Abenavoli and Bellentani, 2013; Colica et al., 2017; Fathalah et al., 2017; Wah Kheong et al., 2017). Thus, NAFLD patients are a target population for silymarin use and may be taking more than the 140-mg dose that is commonly recommended (Zhu et al., 2013). In vitro studies indicate that silymarin, which is a complex of at least seven flavonolignans, inhibits human OATP1B1, OATP1B3, and OATP2B1 $\left(\mathrm{IC}_{50}, 0.3-3.2 \mu \mathrm{M}\right)$ and the canalicular efflux transporter breast cancer-resistance protein (BCRP) $\left(\mathrm{K}_{\mathrm{i}}, 97 \mu \mathrm{M}\right)$ (Deng et al., 2008; Köck et al., 2013).

The objective of this study was to determine the effects of NASH and silymarin, both alone and in combination, on the pharmacokinetics of the clinical OATP probe substrate pitavastatin (Prueksaritanont et al., 2014). Using an established rat model of NASH (Canet et al., 2014), the study was designed to mimic systemic concentrations of silymarin flavonolignans in NAFLD patients taking $700 \mathrm{mg}$ of silymarin. An intravenous administration route was selected to isolate hepatic uptake and efflux processes by bypassing potential variability in oral absorption of pitavastatin and silymarin. Results suggest that the presence of NASH may increase the risk of disease-drug interaction for this OATP-mediated natural product.

\section{Materials and Methods}

Animals. Handling, care, and maintenance of the animals took place in the Program of the Laboratory Animal Resources facility of Washington State University, Spokane, which is accredited by the Association for the Assessment of Laboratory Animal Care International. All animals were maintained in 12-hour light and dark cycles for the duration of the study. The experimental protocol was approved by the Institutional Animal Care and Use Committee at Washington State University. Eight-week-old male Sprague-Dawley rats $(n=16)$ were purchased from Envigo (Huntingdon, Cambridgeshire, UK). Animals were randomly placed in cages (two per cage) with diamond soft-paper bedding and fed either a control diet (cat. no. D518754; Dyets Inc., Bethlehem, PA) or a methionine- and choline-deficient diet (cat. no. D518810; Dyets Inc.). The latter diet is the best rat model for assessing alterations in drug transporters and drug disposition observed in clinical NASH (Canet et al., 2014).

After 8 weeks of diet, animals were anesthetized via intraperitoneal injection of ketamine $(87 \mathrm{mg} / \mathrm{kg}, 1 \mathrm{ml} / \mathrm{kg})$ and xylazine $(13 \mathrm{mg} / \mathrm{kg}$, $1 \mathrm{ml} / \mathrm{kg}$ ). Body temperature was maintained with heated surgical tables and monitored with a rectal thermometer. The trachea was surgically exposed, and a tracheotomy was performed by inserting PE240 tubing (Braintree Scientific, Braintree, MA) into the trachea. Isoflurane $(1.5 \%)$ was administered through a small animal ventilator (Kent Scientific, Torrington, CT) to maintain anesthesia and oxygen through the remainder of the study. The carotid artery and jugular vein were cannulated with PE50 tubing (Braintree Scientific), and the common bile duct was cannulated with PE10 tubing (Braintree Scientific).

Silymarin (cat. no. S0292, lot no. BCBT9170; Sigma Aldrich, St. Louis, MO) was dissolved in sterile saline with $15 \%$ ethanol and $30 \%$ PEG400 by heating to $37^{\circ} \mathrm{C}$ and homogenized with a vortex mixer. Pitavastatin acid, hereafter referred to as pitavastatin (cat. no. ab141958; Abcam, Cambridge, MA), was dissolved in dimethyl sulfoxide (DMSO) and diluted in sterile saline (2\% DMSO final). Silymarin and pitavastatin were prepared fresh daily. The total time for silymarin and pitavastatin administration into the jugular vein was 2 minutes. Silymarin or vehicle was administered first $(10 \mathrm{mg} / \mathrm{kg}$, $1 \mathrm{ml} / \mathrm{kg})$, immediately followed by pitavastatin $(0.5 \mathrm{mg} / \mathrm{kg}, 1 \mathrm{ml} / \mathrm{kg})$.

Blood (approx. $100 \mu \mathrm{l}$ ) was collected into heparinized tubes at 2, 5, $10,20,40,80$, and 120 minutes after silymarin/pitavastatin administration, and bile was collected in 15-minute increments. Plasma was isolated by centrifugation $\left(10,000 \mathrm{~g}\right.$ for 5 minute at $\left.4^{\circ} \mathrm{C}\right)$. Interconversion between the acid and lactone forms of pitavastatin was prevented by acidifying plasma and bile samples with ammonium acetate $(\mathrm{pH}$ 4.2 ) at a volume ratio of 1-15 (acid to biologic sample) immediately after collection (Qi et al., 2013). After the final blood collection, animals were euthanized via exsanguination. Liver and muscle tissues were collected, snap frozen in liquid nitrogen, and stored at $-80^{\circ} \mathrm{C}$ until further analyses.

Western Blot Analysis. Liver tissues were homogenized in NP40 lysis buffer with protease inhibitors (cOmplete Protease Inhibitor Cocktail; Roche, Basel, Switzerland) (100 mg tissue/1 ml buffer) using a TissueLyzer II (Qiagen, Hilden, Germany) with two metal beads (2.4 $\mathrm{mm}$ ) and the following protocol: $30 \mathrm{~Hz}$ for 3 minutes, transfer to ice for 5 minutes, $30 \mathrm{~Hz}$ for 3 minutes. Cellular debris was removed by centrifugation at $15,000 \mathrm{~g}$ for 10 minutes at $4^{\circ} \mathrm{C}$. Ten micrograms of total protein were loaded into 7.5\% SDS-PAGE gels and transferred to polyvinylidene difluoride membranes with a Bio-Rad (Hercules, CA) Trans-Blot Turbo system at 25 V/1.0 A for 30 minutes. Membranes were blocked with 5\% nonfat dry milk in Tris-buffered saline/ Tween 20 and incubated with the following antibody conditions: OATP1B2 (cat. no. 376904, 1:1000; mouse secondary 1:10,000; Santa Cruz Biotechnology, Santa Cruz, CA) and extracellular signaling-regulated kinase (Erk) 1 and 2 (cat. nos. 271269 and 1647; 1:20,000; Santa Cruz Biotechnology). Signal was developed with SuperSignal West Femto (ThermoFisher Scientific, Waltham, MA) and images captured using a Bio-Rad ChemiDoc imager. Densitometry was performed using ImageJ software (National Institutes of Health, Bethesda, MD).

Liquid Chromatography/Mass Spectrometry for Pitavastatin. Quantification of pitavastatin was adapted from published methods (Qi et al., 2013). Chromatographic separation and quantification was performed on a QTRAP 6500 UHPLC-MS/MS system (AB Sciex, Framingham, MA). A gradient of $0.1 \%$ formic acid in water (A) and $0.1 \%$ formic acid in acetonitrile (B) was passed through an HSS T3 column $(1.8 \mu \mathrm{m}, 2.1 \times 50 \mathrm{~mm})$ (Waters Corporation, Waltham, MA) at $50^{\circ} \mathrm{C}$ under the following conditions: $0-1$ minute, ramp from $50 \%$ to $78 \% \mathrm{~B} ; 1-1.5$ minutes $90 \% \mathrm{~B} ; 1.5-2$ minutes, $50 \% \mathrm{~B}$; flow rate $0.5 \mathrm{ml} / \mathrm{min}$. The autosampler was maintained at $4^{\circ} \mathrm{C}$, and the sample injection volume was $2 \mu$ l. The turbo electrospray source was operated in positive ionization mode. The following parameters in multiple reaction monitoring were used for compound detection: pitavastatin, $422.1 \rightarrow 290.1 \mathrm{~m} / z$, declustering potential (DP) $111 \mathrm{~V}$, collision energy (CE) $37 \mathrm{~V}$; pitavastatin lactone (Cayman Chemicals, Ann Arbor, MI), $404.1 \rightarrow 290.1 \mathrm{~m} / z$, DP $111 \mathrm{~V}$, CE $35 \mathrm{~V}$; pitavastatin-d (Toronto $^{-}$ Research Chemicals, Ontario, Canada), $426.9 \rightarrow 293.8 \mathrm{~m} / z$, DP $136 \mathrm{~V}$, CE $41 \mathrm{~V}$; pitavastatin lactone-d d $_{5}$ (Toronto Research Chemicals), 409.1 $\rightarrow 295.2, \mathrm{DP} 81 \mathrm{~V}, \mathrm{CE} 37 \mathrm{~V}$. Plasma and bile samples were processed by mixing $10 \mu \mathrm{l}$ of plasma or $10 \mu \mathrm{l}$ of prediluted bile (20-fold) in water with $50 \mu \mathrm{l}$ of $0.1 \%$ formic acid in water containing $0.24 \mu \mathrm{M}$ pitavastatin- $\mathrm{d}_{5}$ and $1.24 \mu \mathrm{M}$ pitavastatin lactone- $\mathrm{d}_{5}$ deuterated internal standards, followed by addition of $40 \mu \mathrm{l}$ of $0.1 \%$ formic acid in acetonitrile. Samples were homogenized with a vortex mixer and centrifuged at $13,000 \mathrm{~g}$ for 10 minutes at $4^{\circ} \mathrm{C}$. The supernatant was transferred to an autosampler vial for analysis. Liver samples were pulverized with a mortar and pestle under liquid nitrogen. Powdered tissue was mixed with $0.1 \%$ formic acid in water containing $1.2 \mu \mathrm{M}$ pitavastatin- $\mathrm{d}_{5}$ and $6.2 \mu \mathrm{M}$ pitavastatin lactone- $\mathrm{d}_{5}$ deuterated internal standards at a ratio of $50 \mathrm{mg}$ tissue to $100 \mu \mathrm{l}$ of $0.1 \%$ formic acid in water and homogenized with a vortex mixer. The mixture was snap frozen in liquid nitrogen, thawed, homogenized with a vortex mixer, 

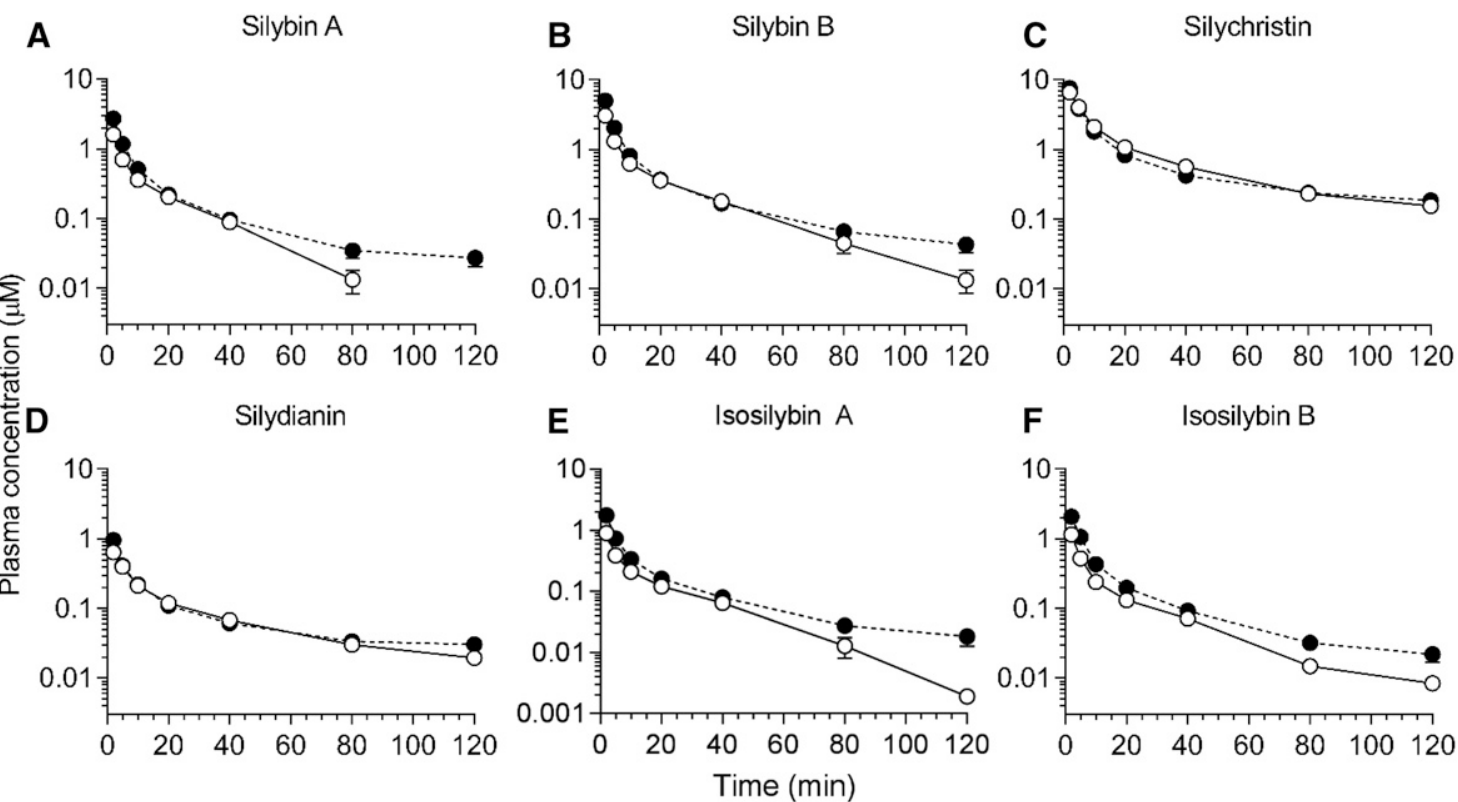

Fig. 1. Average plasma concentration-time profiles for silymarin flavonolignans following intravenous administration of silymarin to rats. Plasma concentration-time profile for silybin A (A), silybin B (B), silychristin (C), silydianin (D), isosilybin A (E), and isosilybin B (F). Open and closed circles denote control and NASH rats, respectively. Circles and error bars represent mean and S.E.M., respectively, of four rats per group.

and centrifuged at $13,000 \mathrm{~g}$ for 10 minutes at $4^{\circ} \mathrm{C}$. Ten microliters of the supernatant were mixed with $80 \mu \mathrm{l}$ of $0.1 \%$ formic acid in acetonitrile, homogenized with a vortex mixer, and centrifuged at $13,000 \mathrm{~g}$ for 10 minutes at $4^{\circ} \mathrm{C}$. The clean supernatant $(70 \mu \mathrm{l})$ was transferred to an autosampler vial for analysis. Quantification of unknowns was accomplished using a calibration curve that was linear from 0.018 to $4.75 \mu \mathrm{M}$ and quality control samples were run periodically to ensure consistent instrument performance.

Liquid Chromatography/Mass Spectrometry for Silymarin Flavonolignans. Quantification of silymarin flavonolignans was adapted from previous methods (Gufford et al., 2014). All purified standards were provided by Dr. Nicholas Oberlies (University of North Carolina at Greensboro, Greensboro, NC). The same UPLCMS/MS system and column used for pitavastatin was used for silymarin flavonolignans. A gradient of $0.1 \%$ formic acid in water (A) and $0.1 \%$ formic acid in methanol (B) at $50^{\circ} \mathrm{C}$ was used under the following conditions: 0 to 0.1 minute, ramp from $5 \%$ to $30 \% \mathrm{~B}$; 0.1-5.5 minutes ramp from $30 \%$ to $53 \%$ B; 5.5-6.5 minutes, $5 \% \mathrm{~B}$; flow rate $0.5 \mathrm{ml} / \mathrm{min}$. The autosampler was maintained at $4^{\circ} \mathrm{C}$, and the sample injection volume was $10 \mu \mathrm{l}$. The turbo electrospray source was operated in the negative ionization mode. The following parameters in multiple reaction monitoring were used for compound detection: silybin A, $480.9 \rightarrow 301.9 \mathrm{~m} / z$, DP $-30 \mathrm{~V}, \mathrm{CE}$ $-26 \mathrm{~V}$; silybin $\mathrm{B}, 480.9 \rightarrow 125.0 \mathrm{~m} / z$, DP $-5 \mathrm{~V}, \mathrm{CE}-34 \mathrm{~V}$; silychristin, $480.9 \rightarrow 325.1 \mathrm{~m} / z$, DP $-5 \mathrm{~V}, \mathrm{CE}-30 \mathrm{~V}$; silydianin, $480.9 \rightarrow 178.9 \mathrm{~m} / z$, DP $-50 \mathrm{~V}, \mathrm{CE}-32 \mathrm{~V}$; isosilybin A, $480.9 \rightarrow$ $125.0 \mathrm{~m} / z$, DP $-70 \mathrm{~V}, \mathrm{CE}-34 \mathrm{~V}$; isosilybin $\mathrm{B}, 480.9 \rightarrow 125.0 \mathrm{~m} / z$, $\mathrm{DP}-10 \mathrm{~V}, \mathrm{CE}-34 \mathrm{~V}$; isosilychristin, $480.9 \rightarrow 463.0, \mathrm{DP}-30 \mathrm{~V}, \mathrm{CE}$ $-22 \mathrm{~V}$; taxifolin, $302.8 \rightarrow 284.9 \mathrm{~m} / z$, DP $-15 \mathrm{~V}, \mathrm{CE}-16 \mathrm{~V}$; all silybin $\beta$-glucuronides, $657.1 \rightarrow 481.1, \mathrm{DP}-25 \mathrm{~V}, \mathrm{CE}-25 \mathrm{~V}$; naringin, $578.9 \rightarrow 270.9, \mathrm{DP}-25 \mathrm{~V}, \mathrm{CE}-44 \mathrm{~V}$. Because chromatographic separation of the silybin A and silybin B forms of the 7-O$\beta$-glucuronides could not be achieved, these compounds were quantified together and referred to as silybinin-7- $O-\beta$-glucuronides. Plasma samples were processed by mixing $20 \mu \mathrm{l}$ of plasma with $50 \mu$ l of naringin $(0.17 \mu \mathrm{M})$ as internal standard, followed by addition of $30 \mu \mathrm{l}$ of $0.1 \%$ formic acid in methanol. Samples were homogenized with a vortex mixer and centrifuged at $13,000 \mathrm{~g}$ for 10 minutes at $4^{\circ} \mathrm{C}$. The clean supernatant $(70 \mu \mathrm{l})$ was transferred to an autosampler vial for analysis. In addition, an aliquot of the silymarin solution administered in the animal study was analyzed using these methods to determine the flavonolignan composition of the dose. Quantification of unknowns was accomplished using a calibration curve that was linear from 0.016 to $4.16 \mu \mathrm{M}$, and quality control samples were run periodically to ensure consistent instrument performance.

Pharmacokinetic Analysis. The pharmacokinetics of the silymarin flavonolignans and pitavastatin were determined via noncompartmental methods using Phoenix WinNonlin (version 7.0; Certara, Princeton, NJ). Area under the plasma concentration-time curve (AUC) from 0 to 120 minutes $\left(\mathrm{AUC}_{0-120 \mathrm{~min}}\right)$ was determined using the logarithmic trapezoidal method. Terminal slope $\left(\lambda_{z}\right)$ was calculated via linear regression of at least the last three data points. AUC from 0 to infinite time $\left(\mathrm{AUC}_{0-\mathrm{inf}}\right)$ was calculated as the sum of $\mathrm{AUC}_{0-120 \text { min }}$ and the ratio of the concentration at 120 minutes to $\lambda_{\mathrm{z}}$. Systemic clearance $(\mathrm{Cl})$ was calculated as the ratio of dose to $\mathrm{AUC}_{0-\text { inf. }}$ Volume of distribution at steady state $\left(\mathrm{V}_{\mathrm{ss}}\right)$ was calculated as the product of $\mathrm{Cl}$ and mean residence time (MRT), where MRT is the ratio of area under the moment curve from zero to infinite time to $\mathrm{AUC}_{0-\text { inf }}$. Terminal half-life $\left(\mathrm{t}_{1 / 2}\right)$ was calculated as the ratio of 0.693 to $\lambda_{\mathrm{z}}$. One of the silymarin-treated NASH animals received the incorrect dose of pitavastatin and was excluded from pitavastatin analyses because the actual dose could not be determined with confidence.

IC $_{50}$ Determination. All cells were maintained at $37^{\circ} \mathrm{C}$ in a humidified $5 \% \mathrm{CO}_{2}$ atmosphere. Human embryonic kidney (HEK) $293 \mathrm{~T} / 17$ cells were seeded at $1.5 \times 10^{5}$ cells per well onto poly-D-lysine-coated 12 -well plates and were transiently transfected with $0.75 \mu \mathrm{g}$ of rat OATP1B2-expressing plasmid (pcDNA5/FRT vector) for 4 hours using jetPRIME (Polyplus Transfection, Illkirch, France). After incubation, the medium was changed to fresh medium with no transfection reagent. Cells were grown for an additional 24 hours before the transport assays were performed. Chinese hamster ovary (CHO) cells stably expressing human OATP1B1 or OATP1B3 were provided by Dr. Bruno Stieger (University of Zurich, Zurich, Switzerland). CHO cells were grown in Dulbecco's modified Eagle's medium (low-glucose) containing $25 \mathrm{mM}$ HEPES, supplemented with $10 \%$ fetal bovine serum, and $50 \mu \mathrm{g} / \mathrm{ml} \mathrm{L}$-proline. Cells were seeded onto 12 -well plates at a density of $1 \times 10^{5}$ cells per well. Transporter expression was induced in CHO cells by addition of $5 \mathrm{mM}$ sodium butyrate to the culture media for 24 hours before transport experiments 
were performed. Cells were washed with prewarmed uptake buffer (135 mM sodium chloride, $1.3 \mathrm{mM}$ HEPES, $2.8 \mathrm{mM}$ D-glucose, $0.5 \mathrm{mM}$ potassium chloride, $0.25 \mathrm{mM}$ calcium dichloride, $0.12 \mathrm{mM}$ magnesium dichloride, $80 \mu \mathrm{M}$ magnesium sulfate, $\mathrm{pH}$ 7.4) three times and allowed to equilibrate in the final wash for 5 minutes. Uptake was initiated by addition of prewarmed transport buffer containing $1 \mu \mathrm{M}$ pitavastatin, and transport was terminated at 3 minutes with ice-cold uptake buffer. Cells were washed quickly three times with ice-cold uptake buffer before being lysed with $110 \mu \mathrm{l}$ of HPLC water. Plates were subjected to a freeze-thaw cycle at $-80^{\circ} \mathrm{C}$ before the wells were scraped and the sample transferred to a microcentrifuge tube. Eighty microliters of the supernatant were mixed with $320 \mu \mathrm{l}$ of acetonitrile containing $0.04 \mu \mathrm{M}$ pitavastatin- $\mathrm{d}_{5}$ and $0.2 \mu \mathrm{M}$ pitavastatin lactone- $\mathrm{d}_{5}$. Samples were homogenized with a vortex mixer and centrifuged at $13,000 \mathrm{~g}$ for 5 minutes at room temperature. The supernatant $(380 \mu \mathrm{l})$ was dried under vacuum at $37^{\circ} \mathrm{C}$, then reconstituted in $50 \mu \mathrm{l}$ of $20 \%$ acetonitrile with $0.1 \%$ formic acid and analyzed for pitavastatin as described above. Transport in wild-type CHO and empty vector-transfected HEK293T/ 17 cells was subtracted from transport in OATP-expressing cells and is presented as percentage of control. Best-fit $\mathrm{IC}_{50}$ values were determined by nonlinear least-squares regression using Phoenix WinNonlin (v. 8.1; Certara, St. Louis, MO) as described previously (Gufford et al., 2014).

Statistical Analysis. All data represent mean \pm S.E.M. Two-way analysis of variance (ANOVA) $P$ values are shown in tables accompanying the respective graph. Tukey's post-hoc test was used to compare groups: a $=P$ value $\leq 0.05$ versus respective vehicle group within each diet group; $\mathrm{b}=P$ value $\leq 0.05$ versus vehicle control; $\mathrm{c}=P$ value $\leq 0.05$ versus silymarin control. Comparisons of silymarin flavonolignans between control and NASH groups were made using the unpaired Student's $t$ test.

\section{Results}

Pharmacokinetics of Silymarin Flavonolignans. The percent extrapolation from $\mathrm{AUC}_{0-120 \text { min }}$ to $\mathrm{AUC}_{120 \text { min-inf }}$ for the silymarin flavonolignans ranged from $0.23 \%$ to $18 \%$. Average $\mathrm{AUC}_{0-\text { inf }}$ of silybin $\mathrm{A}$, silybin $\mathrm{B}$, isosilybin $\mathrm{A}$, and isosilybin $\mathrm{B}$ was 1.5 - to 2.0 -fold higher in the NASH group compared with the control group (Fig. 1; Table 1). Cl of each flavonolignan decreased by $40 \%-69 \%$ in the NASH group compared with the control group (Table 1 ). The terminal $t_{1 / 2}$ of silybin B and isosilybin A increased 1.7- and 1.8-fold, respectively, in the NASH group compared with the control group. $\mathrm{V}_{\mathrm{ss}}$ of each flavonolignan decreased by $40 \%-69 \%$ in the NASH group compared with the control group.

Average $\mathrm{AUC}_{0-120}$ min of silybin A-4"'-O- $\beta$-glucuronide, silybin B-4" $-O-\beta$-glucuronide, and silybinin-7-O- $\beta$-glucuronide was approximately 2-fold higher in the NASH group compared with the control group (Fig. 2). Average total flavonolignan $\mathrm{AUC}_{0-120 \text { min }}$ (flavonolignans plus glucuronides) increased 1.7-fold in the NASH group compared with the control group $(594 \pm 71$ vs. $353 \pm 32 \mathrm{nmol} \times \mathrm{min} / \mathrm{ml}$, mean \pm S.E.M. $)(P<0.001)$.

Pharmacokinetics of Pitavastatin. Pitavastatin lactone was below the detection limit in all samples. The NASH vehicle group showed higher pitavastatin concentrations from 2 to 40 minutes but did not significantly change $\mathrm{AUC}_{0-120}$ min compared with the control vehicle group (Fig. 3, A and B). Average pitavastatin $\mathrm{AUC}_{0-120 \text { min }}$ increased 1.8-fold in the control silymarin group and 2.9-fold in the NASH silymarin group compared with the control vehicle group (Fig. 3B). The amount of pitavastatin in bile was increased by NASH and decreased by silymarin at the early time points (Fig. 4A). Bile flow was increased by NASH and decreased by silymarin at the early time points (Fig. 4B). The total amount of pitavastatin excreted into bile was increased owing to NASH and decreased by silymarin in both control and NASH groups (Fig. 4C). Pitavastatin biliary clearance was decreased approx. $70 \%$ by silymarin in both control and NASH groups (Fig. 4D). The amount of pitavastatin in bile was associated with bile flow (Fig. 4, E and F). At the terminal time point, the total amount of pitavastatin in the liver and the pitavastatin liver-to-plasma ratio were lower in the NASH groups (Fig. 5, A and B). Total pitavastatin in the gastrocnemius (leg muscle) and pitavastatin muscle-to-plasma ratio were not different between the groups (Fig. 5, C and D).

OATP1B2 Protein Expression in NASH and Inhibitory Effects of Silymarin on OATPs. OATP1B2 protein expression decreased in the NASH groups but was not altered 120 minutes after a single dose of silymarin (Fig. 6).

TABLE 1

Silymarin flavonolignan pharmacokinetics

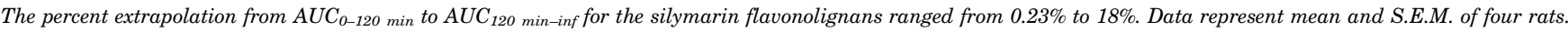

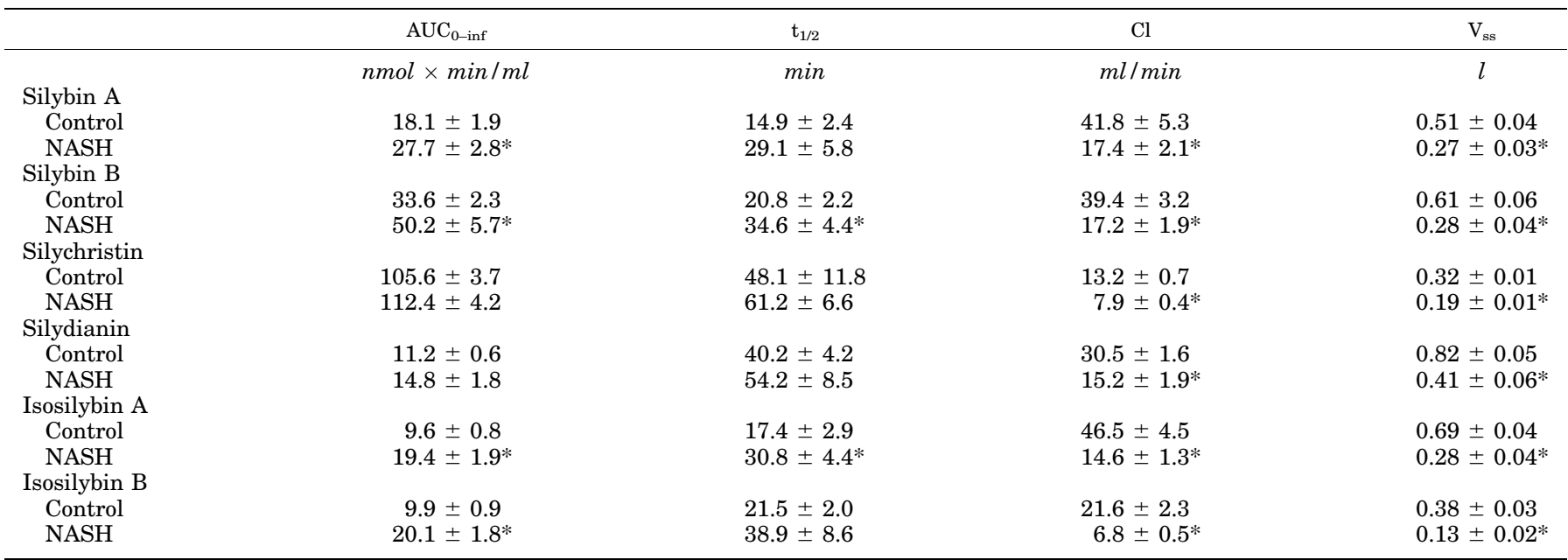

$\mathrm{AUC}_{0-\mathrm{inf}}$, area under the plasma concentration-time curve from time zero to infinite time; $\mathrm{t}_{1 / 2}$, terminal half-life.

$* P$ value $\leq 0.05$ vs. control (unpaired $t$ test). 
A
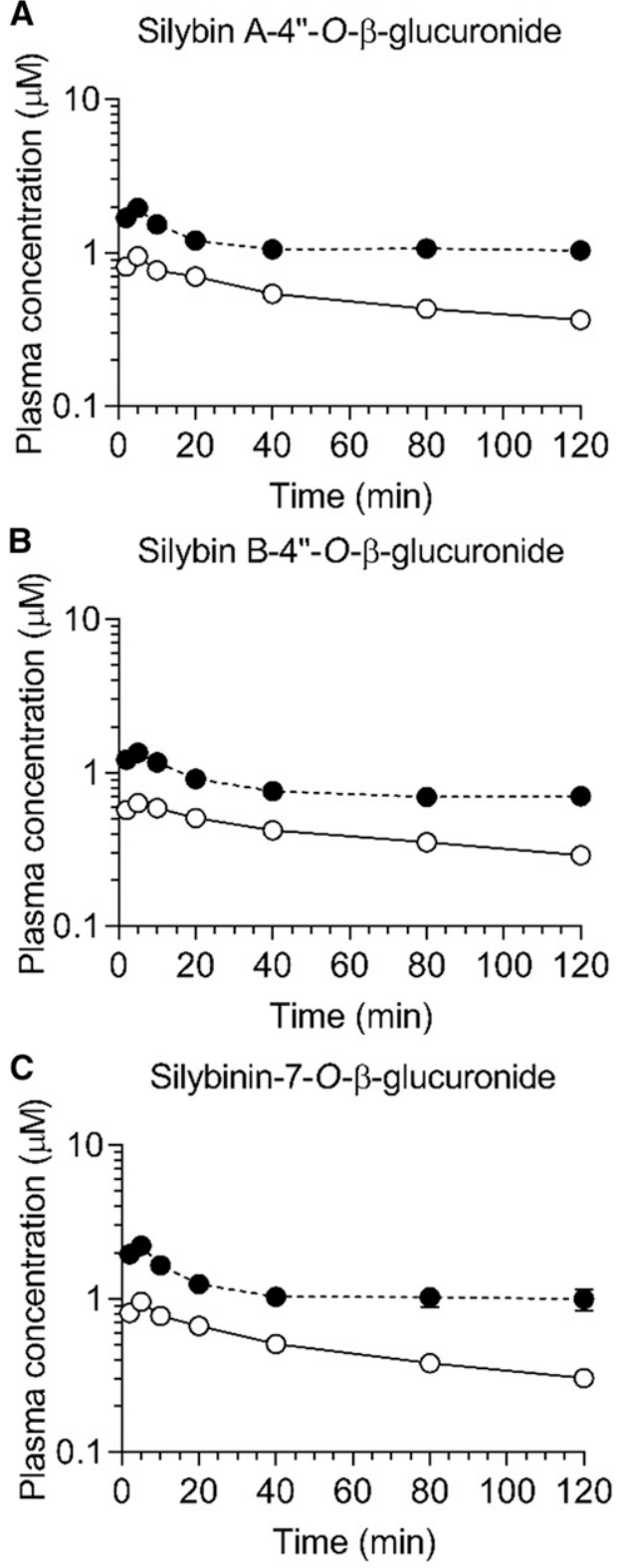

D Silymarin glucuronides $\mathrm{AUC}_{0-120}$

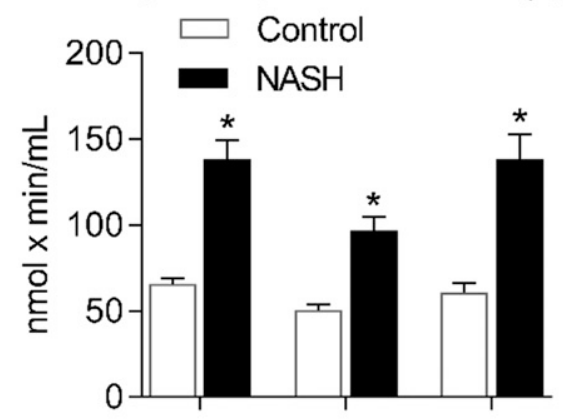

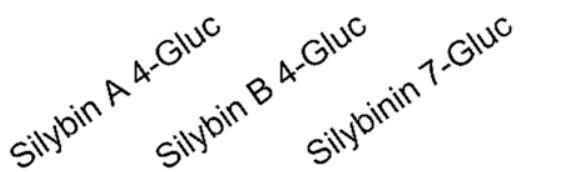

Fig. 2. Average plasma concentration-time profiles for silymarin glucuronides following intravenous administration of silymarin to rats. Plasma concentration-time profile for silybin A- $4{ }^{\prime \prime}-O-\beta$-glucuronide (A), silybin B$4^{\prime \prime}-O-\beta$-glucuronide (B), and silybinin-7-O- $\beta$-glucuronide (C). $\mathrm{AUC}_{0-120 \mathrm{~min}}$

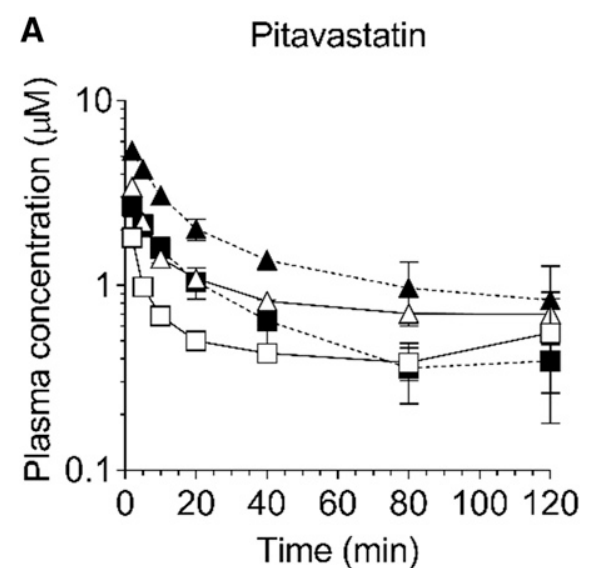

B Pitavastatin $\mathrm{AUC}_{0-120 \mathrm{~min}}$

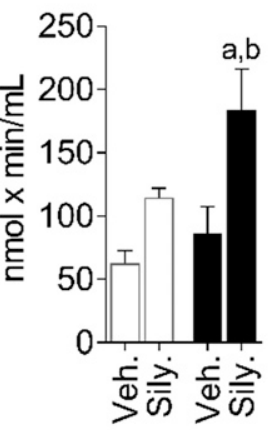

$\square$ Control Vehicle NASH Vehicle

$\triangle$ Control Silymarin $\star$ NASH Silymarin

\begin{tabular}{lc}
\hline 2-way & ${\text { Pitavastatin } \text { AUC }_{0-120 \min }}_{\text {ANOVA }}$ \\
\hline NASH & $<0.05$ \\
Silymarin & $<0.05$ \\
Interaction & 0.24 \\
\hline
\end{tabular}

Fig. 3. Pharmacokinetics of pitavastatin in control and NASH rats with or without silymarin. Average plasma concentration-time profile for pitavastatin (A) and pitavastatin $\mathrm{AUC}_{0-120 \text { min }}$ for each group (B). Circles and error bars represent mean and S.E.M., respectively, of three rats for the NASH silymarin group and of four rats for all other groups. Two-way ANOVA $P$ values are shown in the table. Tukey's post-hoc test: $\mathrm{a}=P$ value $\leq 0.05$ vs. respective vehicle group within each diet group; $\mathrm{b}=P$ value $\leq 0.05$ vs. vehicle control.

Silymarin inhibited rat OATP1B2- and human OATP1B1- and OATP1B3-mediated uptake of pitavastatin, with $\mathrm{IC}_{50}$ values of 13.8, 6.5, and $12.7 \mu \mathrm{M}$, respectively (Fig. 7).

\section{Discussion}

A previous clinical study reported no pharmacokinetic interaction between silymarin and an OATP substrate (rosuvastatin) in healthy subjects (Deng et al., 2008). We hypothesized that the presence of NASH may increase the risk for an OATP-mediated silymarin-drug interaction compared with a healthy condition owing to decreased hepatic OATP protein expression and increased systemic silymarin flavonolignan concentrations (Schrieber et al., 2008; Clarke et al., 2017). The present study was designed to address this hypothesis by mimicking key aspects of a clinical NAFLD scenario in an established rodent model of human NASH (Clarke et al., 2014).

Pitavastatin has been proposed as an effective probe drug for OATP1B function because it is minimally metabolized and is primarily dependent on OATPs for hepatic sinusoidal uptake (Hirano et al., 2004, 2005; Prueksaritanont et al., 2014, 2017). Although we confirmed that silymarin inhibits rat OATP1B2, a limitation of using a rodent model to test our hypothesis is that other rodent orthologs beyond OATP1B2 (e.g., OATP1A isoforms) may have been contributing to the

for each glucuronide (D). Open and closed circles (A-C) denote control and NASH rats, respectively. Circles and error bars represent mean and S.E.M., respectively, of four rats per group. $* P$ value $\leq 0.05$ vs. control (unpaired $t$ test). 
A

Bile

B

Bile flow


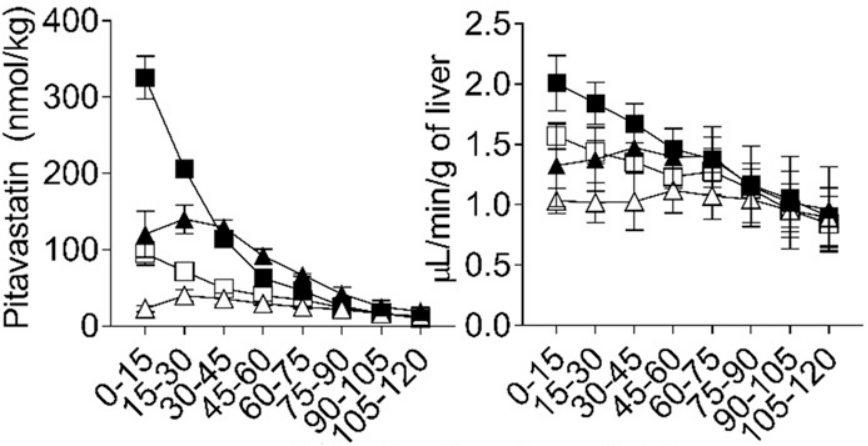

Collection time frame $(\mathrm{min})$

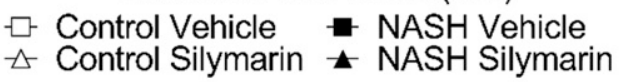

C

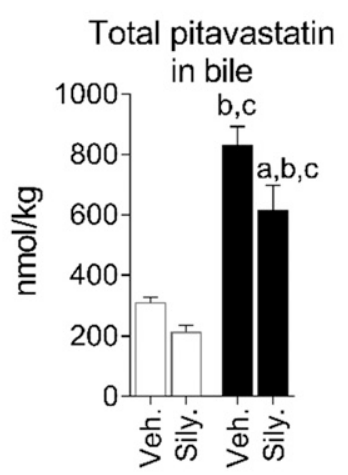

D
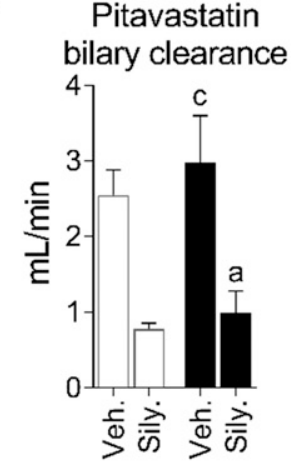

Control $\square \mathrm{NASH}$

\begin{tabular}{lcc}
\hline $\begin{array}{l}\text { 2-way } \\
\text { ANOVA }\end{array}$ & $\begin{array}{c}\text { Total pitavastatin } \\
\text { in bile }\end{array}$ & $\begin{array}{c}\text { Pitavastatin } \\
\text { biliary } \\
\text { clearance }\end{array}$ \\
\hline NASH & $<0.05$ & 0.45 \\
Silymarin & $<0.05$ & $<0.05$ \\
Interaction & 0.26 & 0.80 \\
\hline
\end{tabular}

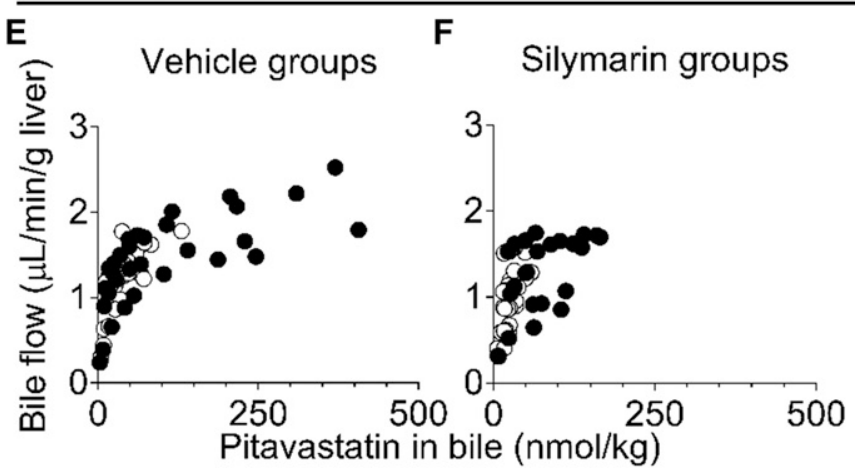

Fig. 4. Biliary excretion of pitavastatin in control and NASH rats with or without silymarin. Amount of pitavastatin recovered in bile for each collection interval (A). Bile flow for each collection interval (B). Total pitavastatin recovered in bile (C) and pitavastatin biliary clearance (D). Data in (A-D) represent mean and S.E.M. of three rats for the NASH silymarin group and of four rats for all other groups. Relationship between bile flow and amount of pitavastatin for the vehicle-treated (E) and silymarin-treated (F) groups. Open circles and closed circles denote individual control and NASH rats, respectively. Two-way ANOVA $P$ values are shown in the tables below the respective datasets. Tukey's posthoc test: $\mathrm{a}=P$ value $\leq 0.05$ vs. respective vehicle group within each diet group; $\mathrm{b}=P$ value $\leq 0.05$ vs. vehicle control; $\mathrm{c}=P$ value $\leq 0.05$ vs. silymarin control.
A Liver

B Liver to plasma ratio
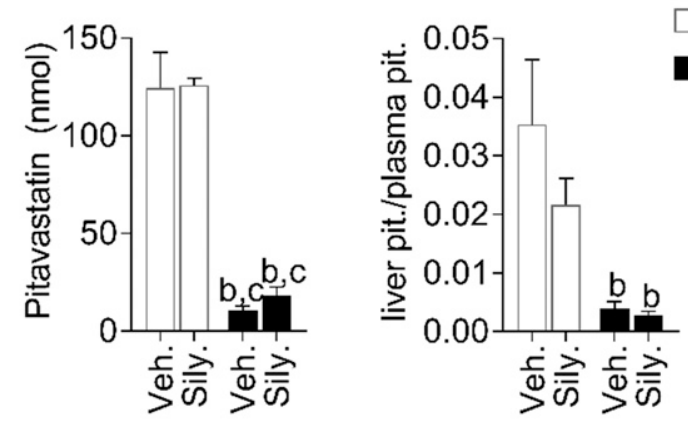

\begin{tabular}{lcc}
\hline $\begin{array}{l}\text { 2-way } \\
\text { ANOVA }\end{array}$ & Liver & $\begin{array}{c}\text { Liver to } \\
\text { plasma ratio }\end{array}$ \\
\hline NASH & $<\mathbf{0 . 0 5}$ & $<\mathbf{0 . 0 5}$ \\
Silymarin & 0.67 & 0.28 \\
Interaction & 0.79 & 0.36 \\
\hline
\end{tabular}

C

\section{Gastrocnemius \\ Muscle to plasma ratio}
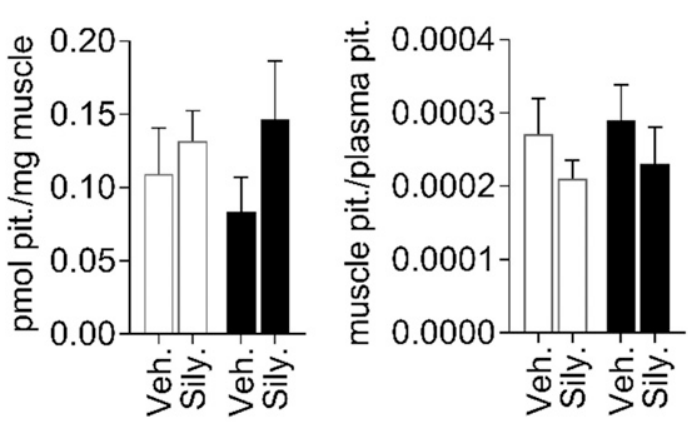

$\square$ Control

- $\mathrm{NASH}$

\begin{tabular}{lcc}
\hline $\begin{array}{l}\text { 2-way } \\
\text { ANOVA }\end{array}$ & Gastroc. & $\begin{array}{c}\text { Muscle to } \\
\text { plasma ratio }\end{array}$ \\
\hline NASH & 0.84 & 0.68 \\
Silymarin & 0.16 & 0.20 \\
Interaction & 0.49 & 0.99 \\
\hline
\end{tabular}

Fig. 5. Tissue concentrations and tissue-to-plasma ratios of pitavastatin in control and NASH rats with or without silymarin. Liver pitavastatin content (A), pitavastatin liver-to-plasma ratio (B), gastrocnemius (leg muscle) pitavastatin concentrations (C), and pitavastatin muscle-toplasma ratio (D) at 2 hours post-dose. Bars and error bars represent mean and S.E.M., respectively, of three rats for the NASH silymarin group and of four rats for all other groups. Two-way ANOVA $P$ values are shown in the table. Tukey's post-hoc test: $\mathrm{b}=P$ value $\leq 0.05$ vs. vehicle control; $\mathrm{c}$ $=P$ value $\leq 0.05$ vs. silymarin control

disposition of pitavastatin (Chang et al., 2019). Silymarin increased pitavastatin exposure in both the control and NASH groups, suggesting that silymarin doses at or above $700 \mathrm{mg}$ may precipitate OATP-mediated drug interactions. These data are noteworthy because higher doses of silymarin, as well as milk thistle formulations designed to improve flavonolignan relative oral bioavailability, are increasingly available on the market. In addition, the combination of NASH and silymarin had the greatest effect on pitavastatin exposure, suggesting that the combined effects of NASH-mediated decrease in the protein expression of and silymarin-mediated inhibition of hepatic OATPs may place NASH patients at the highest risk for this OATP-mediated silymarin-drug interaction. These data are consistent with a previous study indicating that a double hit on hepatic 


\section{OATP1B2 protein}

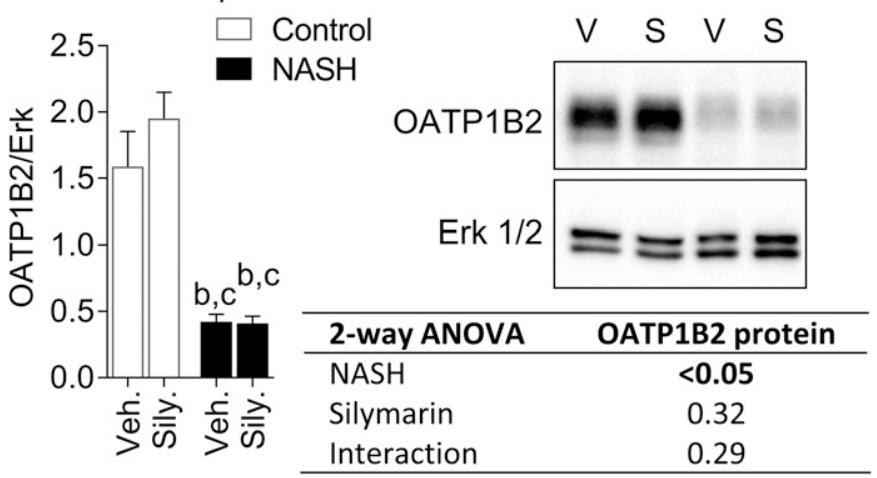

Fig. 6. OATP1B2 expression in control and NASH rats. Representative Western blot for OATP1B2 and the loading control, extracellular signalregulated kinase (Erk) 1/2. Bars and error bars represent mean and S.E.M., respectively, of four rats per group. Two-way ANOVA $P$ values are shown in the table. Tukey's post-hoc test: $\mathrm{b}=P$ value $\leq 0.05$ vs. vehicle control; $\mathrm{c}=P$ value $\leq 0.05$ vs. silymarin control.

OATP-mediated uptake has a greater effect on drug exposure than each hit alone and provide additional impetus for careful evaluation of multifactorial effects on pharmacokinetics (Clarke et al., 2014).

In addition to sinusoidal uptake and efflux, the current data suggest that pitavastatin biliary excretion is affected predominately by bile flow. In the NASH groups, increased bile flow probably contributed to increased pitavastatin biliary excretion. NASH alone did not increase pitavastatin biliary clearance, potentially the result of variability in these data caused by capturing fewer than five half-lives. In the silymarin-treated groups, decreased bile flow probably contributed to decreased pitavastatin biliary excretion. These opposing effects of NASH and silymarin on bile flow contrast with their combined effects on pitavastatin systemic exposure. Interestingly, the effect of bile flow on pitavastatin in bile is not a linear relationship, such that at lower pitavastatin concentrations, the relationship has a steeper slope compared with the relationship at higher concentrations. The silymarin-mediated leftward shift in the NASH pitavastatin data (Fig. $4 \mathrm{E}$ vs. Fig. $4 \mathrm{~F}$ ) suggest that the previously reported increase in BCRP protein expression in NASH potentially plays a role in producing high pitavastatin biliary concentrations (Hardwick et al., 2011; Toth et al., 2018). The silymarin-mediated decrease in bile flow observed in the present study is inconsistent with a previous report showing that intraperitoneal administration of silymarin increased bile flow (Crocenzi et al., 2000). The reason for this discrepancy is unclear but may be related to the strain of rats used (Wistar vs. Sprague Dawley) or route of silymarin administration (intraperitoneal vs. intravenous). Silymarinmediated inhibition of BCRP is not expected to contribute to altered biliary disposition of pitavastatin because of the high $\mathrm{K}_{\mathrm{i}}$ reported against BCRP $(97 \mu \mathrm{M})$ (Deng et al., 2008). Collectively, these data indicate that opposing effects of NASH and silymarin on bile flow influence pitavastatin biliary disposition in a rodent model.

The previous clinical study that found no pharmacokinetic interaction between silymarin ( $140 \mathrm{mg}$ three times daily) and an OATP/BCRP substrate, rosuvastatin (10 mg single dose), has a number of limitations, some of which arose after the study was completed over a decade ago (Deng et al., 2008).
A

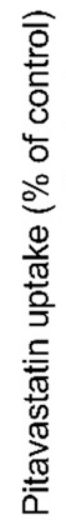
140
120
100
80
60
4
2

B

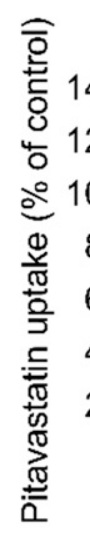

C



Fig. 7. Silymarin inhibition of OATP1B2-, OATP1B1-, and OATP1B3mediated uptake of pitavastatin. IC $_{50}$ curves for OATP1B2 (A), OATP1B1 (B), and OATP1B3 (C) were obtained by measuring uptake of $1 \mu \mathrm{M}$ pitavastatin for 3 minutes in the absence or presence of increasing silymarin concentrations. Symbols and error bars represent mean and S.E.M., respectively, of six replicates. Curves denote model-generated best fits to the data. $\mathrm{IC}_{50}$ values represent model-generated best-fit estimates \pm S.E.'s of the estimates. 
In addition to the limitations discussed by the authors of that study, many studies performed after 2008 have used silymarin doses greater than $140 \mathrm{mg}$ three times daily (e.g., $700 \mathrm{mg}$ three times daily) and/or formulations that increase flavonolignan plasma concentrations and relative oral bioavailability (Kumar et al., 2014; Poruba et al., 2015; Liang et al., 2018). These doses and formulations that can produce higher plasma concentrations, coupled with the higher plasma concentrations observed in NAFLD patients, suggest that silymarinmediated OATP substrate interactions may occur in this multifactorial scenario. Although we were able to match maximum silybin A plasma concentrations reported for NAFLD and hepatitis $\mathrm{C}$ virus-infected patients taking $560-700 \mathrm{mg}$ of silymarin (present study: 1.7-2.7 $\mu \mathrm{M}$; clinical studies: $0.9-4.2 \mu \mathrm{M}$ ), an important limitation of our study is that intravenous administration of silymarin produces higher plasma concentrations of the other silymarin flavonolignans than are typically observed after oral administration (Hawke et al., 2010; Schrieber et al., 2011; Fried et al., 2012; Köck et al., 2013). Without $\mathrm{IC}_{50}$ values for each of the flavonolignans it is difficult to determine how each flavonolignan may be contributing to the inhibition of OATPs and increased pitavastatin systemic exposure. It remains to be determined whether these current preclinical data and the limitations of the previous clinical pharmacokinetic interaction study necessitate a re-evaluation of the risk for this natural product-drug interaction.

One of the challenges facing pharmacokinetic natural product-drug interaction research is the large variability in the composition of commercially available products. The Center of Excellence for Natural Product-Drug Interaction Research (NaPDI Center) was created to address this challenge and others by developing Recommended Approaches for studying pharmacokinetic natural product-drug interactions (Paine et al., 2018). In the present study, the composition of the silymarin product was characterized and compared with previously published data on the composition of other silymarin products. The product used in this study contained less than $50 \%$ silybin A and silybin B (Fig. 8), which contrasts with a silymarin capsule and another lot of silymarin from the same source, both of which contained more than $55 \%$ silybin A and silybin B (Wen et al., 2008). In addition, the silymarin product used in the current study contained more than $30 \%$ silychristin, whereas all other products contained approx. $20 \%$ silychristin (Wen et al., 2008; Hawke et al., 2010). The $\mathrm{IC}_{50}$ values for silymarin-mediated inhibition of pitavastatin uptake by human OATP1B1 $(6.5 \mu \mathrm{M})$ and OATP1B3 $(12.7 \mu \mathrm{M})$ were higher than those previously reported using estrone-3sulfate as the probe substrate (1.3 and $2.2 \mu \mathrm{M}$, respectively) (Köck et al., 2013). Another group, who used a mix of disuccinated silybin $\mathrm{A}$ and silybin $\mathrm{B}$, reported an $\mathrm{IC}_{50}$ of $3.3 \mu \mathrm{M}$ for OATP1B1 and a $\mathrm{K}_{\mathrm{i}}$ of $5 \mu \mathrm{M}$ for OATP1B3 using estradiol $17 \beta$-glucuronide as the probe substrate (Wlcek et al., 2013). These differences may reflect different compositions of the silymarin products and/or the substrate used. In addition, the higher percentage of silychristin in the current study may have contributed to the higher $\mathrm{IC}_{50}$ values, as previous data suggest that silychristin may be a less potent OATP inhibitor (Köck et al., 2013). Unfortunately, a direct comparison between the previously published and current $\mathrm{IC}_{50}$ values cannot be made because the composition of the product used in the previous study was not reported and the probe substrates were different (Köck

\section{Flavonolignan constituents in silymarin}

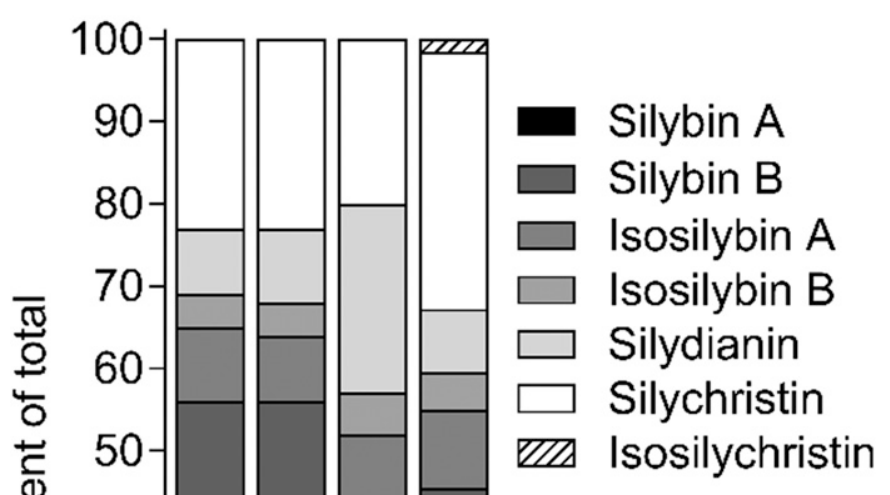

Fig. 8. Flavonolignan constituents in the silymarin used in this study and others. Silybin A, silybin B, isosilybin A, isosilybin B, silydianin, and silychristin expressed as percent of total flavonolignan composition. Composition of silymarin powder purchased from Sigma-Aldrich, and used in the current study, was compared with that in capsules used in two separate pharmacokinetic studies and from another lot of silymarin powder purchased from Sigma-Aldrich measured by Wen et al. (2008), Hawke et al. (2010).

et al., 2013). These data highlight the importance of characterizing the constituent composition of a given natural product and its interaction with the probe drug of interest in pharmacokinetic natural product-drug interaction studies.

In conclusion, these data provide further evidence that double hit pharmacokinetic interactions can have a greater effect on drug exposure than each hit alone. This may be important for patients with chronic diseases such as NASH, because these patients are often subject to polypharmacy to manage and/or treat comorbidities. In addition, patients afflicted with multiple chronic diseases would be more inclined to take botanical dietary supplements, potentially placing them at greatest risk for adverse drug effects (Dickinson and MacKay, 2014; Patel et al., 2017; Chalasani et al., 2018). 


\section{Acknowledgments}

We gratefully acknowledge Dr. Bruno Stieger from the University of Zurich for providing the OATP1B1 and OATP1B3 expressing CHO cells used in these experiments. We are grateful to Dr. Nicholas Oberlies from the University of North Carolina at Greensboro, who provided the silymarin flavonolignan and glucuronide standards.

\section{Authorship Contributions}

Participated in research design: Montonye, Hagenbuch, Paine, Clarke.

Conducted experiments: Montonye, Arman, Lynch, Clarke.

Performed data analysis: Montonye, Arman, Tian, Lynch, Hagenbuch, Clarke.

Wrote or contributed to the writing of the manuscript: Montonye, Arman, Tian, Lynch, Hagenbuch, Paine, Clarke.

\section{References}

Abenavoli L and Bellentani S (2013) Milk thistle to treat non-alcoholic fatty liver disease: dream or reality? Expert Rev Gastroenterol Hepatol 7:677-679.

Abenavoli L, Capasso R, Milic N, and Capasso F (2010) Milk thistle in liver diseases: past, present, future. Phytother Res 24:1423-1432.

Ali I, Slizgi JR, Kaullen JD, Ivanovic M, Niemi M, Stewart PW, Barritt AS IV, and Brouwer KLR (2017) Transporter-mediated alterations in patients with NASH increase systemic and hepatic exposure to an OATP and MRP2 substrate. Clin Pharmacol Ther 104:749-756.

Canet MJ, Hardwick RN, Lake AD, Dzierlenga AL, Clarke JD, and Cherrington NJ (2014) Modeling human nonalcoholic steatohepatitis-associated changes in drug transporter expression using experimental rodent models. Drug Metab Dispos 42:586-595.

Chalasani N, Younossi Z, Lavine JE, Charlton M, Cusi K, Rinella M, Harrison SA, Brunt EM, and Sanyal AJ (2018) The diagnosis and management of nonalcoholic fatty liver disease: practice guidance from the American Association for the Study of Liver Diseases. Hepatology 67:328-357.

Chang JH, Zhang X, Messick K, Chen Y-C, Chen E, Cheong J, and Ly J (2019) Unremarkable impact of Oatp inhibition on the liver concentration of fluvastatin, lovastatin and pitavastatin in wild-type and Oatp1a/1b knockout mouse. Xenobiotica 49:602-610.

Clarke JD, Hardwick RN, Lake AD, Canet MJ, and Cherrington NJ (2014) Experimental nonalcoholic steatohepatitis increases exposure to simvastatin hydroxy acid by decreasing hepatic organic anion transporting polypeptide expression. $J$ Pharmacol Exp Ther 348:452-458.

Clarke JD, Novak P, Lake AD, Hardwick RN, and Cherrington NJ (2017) Impaired $\mathrm{N}$-linked glycosylation of uptake and efflux transporters in human non-alcoholic fatty liver disease. Liver Int 37:1074-1081.

Colica C, Boccuto L, and Abenavoli L (2017) Silymarin: an option to treat nonalcoholic fatty liver disease. World J Gastroenterol 23:8437-8438.

Crocenzi FA, Pellegrino JM, Sánchez Pozzi EJ, Mottino AD, Garay EA, and Roma MG (2000) Effect of silymarin on biliary bile salt secretion in the rat. Biochem Pharmacol 59:1015-1022.

Deng JW, Shon J-H, Shin H-J, Park S-J, Yeo C-W, Zhou H-H, Song I-S, and Shin J-G (2008) Effect of silymarin supplement on the pharmacokinetics of rosuvastatin. Pharm Res 25:1807-1814.

Dickinson A and MacKay D (2014) Health habits and other characteristics of dietary supplement users: a review. Nutr $J$ 13:14.

Dzierlenga AL, Clarke JD, Hargraves TL, Ainslie GR, Vanderah TW, Paine MF, and Cherrington NJ (2015) Mechanistic basis of altered morphine disposition in nonalcoholic steatohepatitis. J Pharmacol Exp Ther 352:462-470.

Fathalah WF, Abdel Aziz MA, Abou El Soud NH, and El Raziky MES (2017) High dose of silymarin in patients with decompensated liver disease: a randomized controlled trial. J Interferon Cytokine Res 37:480-487.

Ferslew BC, Johnston CK, Tsakalozou E, Bridges AS, Paine MF, Jia W, Stewart PW, Barritt AS IV, and Brouwer KLR (2015) Altered morphine glucuronide and bile acid disposition in patients with nonalcoholic steatohepatitis. Clin Pharmacol Ther 97:419-427.

Fried MW, Navarro VJ, Afdhal N, Belle SH, Wahed AS, Hawke RL, Doo E, Meyers $\mathrm{CM}$, and Reddy KR; Silymarin in NASH and C Hepatitis (SyNCH) Study Group (2012) Effect of silymarin (milk thistle) on liver disease in patients with chronic hepatitis C unsuccessfully treated with interferon therapy: a randomized controlled trial. JAMA 308:274-282.

Gufford BT, Chen G, Lazarus P, Graf TN, Oberlies NH, and Paine MF (2014) Identification of diet-derived constituents as potent inhibitors of intestinal glucuronidation. Drug Metab Dispos 42:1675-1683.

Hardwick RN, Ferreira DW, More VR, Lake AD, Lu Z, Manautou JE, Slitt AL, and Cherrington NJ (2013) Altered UDP-glucuronosyltransferase and sulfotransferase expression and function during progressive stages of human nonalcoholic fatty liver disease. Drug Metab Dispos 41:554-561.

Hardwick RN, Fisher CD, Canet MJ, Scheffer GL, and Cherrington NJ (2011) Variations in ATP-binding cassette transporter regulation during the progression of human nonalcoholic fatty liver disease. Drug Metab Dispos 39:2395-2402.
Hardwick RN, Fisher CD, Street SM, Canet MJ, and Cherrington NJ (2012) Molecular mechanism of altered ezetimibe disposition in nonalcoholic steatohepatitis. Drug Metab Dispos 40:450-460.

Hawke RL, Schrieber SJ, Soule TA, Wen Z, Smith PC, Reddy KR, Wahed AS, Belle SH, Afdhal NH, Navarro VJ, et al.; SyNCH Trial Group (2010) Silymarin ascending multiple oral dosing phase I study in noncirrhotic patients with chronic hepatitis C. J Clin Pharmacol 50:434-449.

Hirano M, Maeda K, Matsushima S, Nozaki Y, Kusuhara H, and Sugiyama Y (2005) Involvement of BCRP (ABCG2) in the biliary excretion of pitavastatin. Mol Pharmacol 68:800-807.

Hirano M, Maeda K, Shitara Y, and Sugiyama Y (2004) Contribution of OATP2 (OATP1B1) and OATP8 (OATP1B3) to the hepatic uptake of pitavastatin in humans. J Pharmacol Exp Ther 311:139-146.

Köck K, Xie Y, Hawke RL, Oberlies NH, and Brouwer KLR (2013) Interaction of silymarin flavonolignans with organic anion-transporting polypeptides. Drug Metab Dispos 41:958-965.

Kumar N, Rai A, Reddy ND, Raj PV, Jain P, Deshpande P, Mathew G, Kutty NG, Udupa N, and Rao CM (2014) Silymarin liposomes improves oral bioavailability of silybin besides targeting hepatocytes, and immune cells. Pharmacol Rep 66: 788-798.

Liang J, Liu Y, Liu J, Li Z, Fan Q, Jiang Z, Yan F, Wang Z, Huang P, and Feng N (2018) Chitosan-functionalized lipid-polymer hybrid nanoparticles for oral delivery of silymarin and enhanced lipid-lowering effect in NAFLD. J Nanobiotechnology 16:64.

Paine MF, Shen DD, and McCune JS (2018) Recommended approaches for pharmacokinetic natural product-drug interaction research: a NaPDI center commentary. Drug Metab Dispos 46:1041-1045.

Patel PJ, Hayward KL, Rudra R, Horsfall LU, Hossain F, Williams S, Johnson T, Brown NN, Saad N, Clouston AD, et al. (2017) Multimorbidity and polypharmacy in diabetic patients with NAFLD: implications for disease severity and management. Medicine (Baltimore) 96:e6761.

Poruba M, Kazdová L, Oliyarnyk O, Malinská H, Matusková Z, Tozzi di Angelo I, Skop V, and Vecera R (2015) Improvement bioavailability of silymarin ameliorates severe dyslipidemia associated with metabolic syndrome. Xenobiotica 45:751-756.

Prueksaritanont T, Chu X, Evers R, Klopfer SO, Caro L, Kothare PA, Dempsey C, Rasmussen S, Houle R, Chan G, et al. (2014) Pitavastatin is a more sensitive and selective organic anion-transporting polypeptide $1 \mathrm{~B}$ clinical probe than rosuvastatin. Br J Clin Pharmacol 78:587-598.

Prueksaritanont T, Tatosian DA, Chu X, Railkar R, Evers R, Chavez-Eng C, Lutz R, Zeng W, Yabut J, Chan GH, et al. (2017) Validation of a microdose probe drug cocktail for clinical drug interaction assessments for drug transporters and CYP3A. Clin Pharmacol Ther 101:519-530.

Qi X, Ding L, Wen A, Zhou N, Du X, and Shakya S (2013) Simple LC-MS/MS methods for simultaneous determination of pitavastatin and its lactone metabolite in human plasma and urine involving a procedure for inhibiting the conversion of pitavastatin lactone to pitavastatin in plasma and its application to a pharmacokinetic study. J Pharm Biomed Anal 72:8-15.

Schrieber SJ, Hawke RL, Wen Z, Smith PC, Reddy KR, Wahed AS, Belle SH, Afdhal NH, Navarro VJ, Meyers CM, et al. (2011) Differences in the disposition of silymarin between patients with nonalcoholic fatty liver disease and chronic hepatitis C. Drug Metab Dispos 39:2182-2190.

Schrieber SJ, Wen Z, Vourvahis M, Smith PC, Fried MW, Kashuba ADM, and Hawke RL (2008) The pharmacokinetics of silymarin is altered in patients with hepatitis C virus and nonalcoholic Fatty liver disease and correlates with plasma caspase-3/7 activity. Drug Metab Dispos 36:1909-1916.

Smith T, Kawa K, Eckl V, Morton C, and Stredney R (2018) Retail sales of herbal supplements in US increase by $8.5 \%$ in 2017 , topping $\$ 8$ billion. HerbalGram (Sept 11, 2018) 62-71.

Toth EL, Li H, Dzierlenga AL, Clarke JD, Vildhede A, Goedken M, and Cherrington NJ (2018) Gene-by-environment interaction of $\mathrm{Bcrp}^{-/-}$and methionine- and cholinedeficient diet-induced nonalcoholic steatohepatitis alters SN-38 disposition. Drug Metab Dispos 46:1478-1486.

Wah Kheong C, Nik Mustapha NR, and Mahadeva S (2017) A randomized trial of silymarin for the treatment of nonalcoholic steatohepatitis. Clin Gastroenterol Hepatol 15:1940-1949.e8.

Wen Z, Dumas TE, Schrieber SJ, Hawke RL, Fried MW, and Smith PC (2008) Pharmacokinetics and metabolic profile of free, conjugated, and total silymarin flavonolignans in human plasma after oral administration of milk thistle extract. Drug Metab Dispos 36:65-72.

Wlcek K, Koller F, Ferenci P, and Stieger B (2013) Hepatocellular organic aniontransporting polypeptides (OATPs) and multidrug resistance-associated protein 2 (MRP2) are inhibited by silibinin. Drug Metab Dispos 41:1522-1528.

Zhu H-J, Brinda BJ, Chavin KD, Bernstein HJ, Patrick KS, and Markowitz JS (2013) An assessment of pharmacokinetics and antioxidant activity of free silymarin flavonolignans in healthy volunteers: a dose escalation study. Drug Metab Dispos 41:1679-1685.

Address correspondence to: Dr. John D. Clarke, Department of Pharmaceutical Sciences, Washington State University, Pharmaceutical and Biomedical Sciences Building, 412 East Spokane Falls Blvd, Spokane, WA 99202. E-mail: j.clarke@wsu.edu 